\title{
Radiation-induced growth and isothermal decay of infrared- stimulated luminescence from feldspar
}

Benny Guralnik, Bo Li, Mayank Jain, Reuven Chen, Richard B. Paris, Andrew S. Murray, Sheng-Hua Li, Vasilis Pagonis, Pierre G. Valla, Frédéric Herman

This is the accepted manuscript (C) 2015, Elsevier Licensed under the Creative Commons Attribution-NonCommercial-NoDerivatives 4.0 International http://creativecommons.org/licenses/by-nc-nd/4.0/

\section{(c) EY-NC-ND}

The published article is available from doi:

http://dx.doi.org/10.1016/j.radmeas.2015.02.011 


\section{Radiation-induced growth and isothermal decay of infrared- stimulated luminescence from feldspar}

Benny Guralnik ${ }^{a,}$, , Bo Li ${ }^{b}$, Mayank Jain ${ }^{c}$, Reuven Chen ${ }^{d}$, Richard B. Paris ${ }^{e}$, Andrew S. Murray ${ }^{\dagger}$, Sheng-Hua $\mathrm{Li}^{g}{ }^{\mathrm{g}}$, Vasilis Pagonis ${ }^{\mathrm{h}}$, Pierre G. Valla ${ }^{\mathrm{i}}$, Frédéric Herman ${ }^{i}$

${ }^{a}$ Department of Earth Sciences, ETH-Zurich, 8092 Zurich, Switzerland

${ }^{b}$ Centre for Archaeological Science, School of Earth and Environmental Sciences, University of Wollongong, Wollongong, NSW 2522, Australia.

${ }^{\mathrm{C}}$ Centre for Nuclear Technologies, Technical University of Denmark, DTU Risø campus, DK 4000 Roskilde, Denmark.

${ }^{d}$ Raymond and Beverly Sackler School of Physics and Astronomy, Tel-Aviv University, 69978 TelAviv, Israel.

${ }^{\text {e }}$ University of Abertay, Dundee, DD1 1HG, UK.

${ }^{\dagger}$ Nordic Laboratory for Luminescence Dating, Department of Geoscience, Aarhus University, DTU Risø Campus, Denmark

${ }^{9}$ Physics Department, McDaniel College, Westminster, MD 21157, USA.

${ }^{\mathrm{h}}$ Department of Earth Sciences, The University of Hong Kong, Pokfulam Road, Hong Kong, China. ' Institute of Earth Surface Dynamics, University of Lausanne, Geopolis, 1015 Lausanne, Switzerland. * Corresponding author. Present address: Netherlands Centre for Luminescence Dating, Droevendaalsesteeg 4, 6708 PB Wageningen, The Netherlands. E-mail address: benny.guralnik@gmail.com (B. Guralnik)

\section{Abstract}

Optically stimulated luminescence (OSL) ages can determine a wide range of geological events or processes, such as the timing of sediment deposition, the exposure of a rock surface, or the cooling of bedrock. The accuracy of OSL dating critically depends on our capability to describe the growth and decay of laboratoryregenerated luminescence signals. Here we review a selection of common models describing the response of infrared stimulated luminescence (IRSL) of feldspar to constant radiation and temperature as administered in the laboratory. We use this opportunity to introduce a general-order kinetic model that successfully captures the behaviour of different materials and experimental conditions with a minimum of model parameters, and thus appears suitable for future application and validation in natural environments. Finally, we evaluate all the presented models by their ability to accurately describe a recently published feldspar multi-elevated temperature post-IR IRSL (MET-pIRIR) dataset, and highlight each model's strengths and shortfalls. 


\section{Introduction}

Optically stimulated luminescence (OSL) dating of feldspar, commonly utilising stimulation with infrared (IR) light and hence termed IRSL, is a group of methods enabling the determination of depositional ages of middle to late Quaternary sediments (Hütt et al., 1988; Buylaert et al., 2012; Li et al., 2014). More recently, the geological applications of feldspar IRSL have been extended to surface exposure dating (Sohbati et al., 2011) and low-temperature thermochronology (Guralnik et al., under review). In addition to the chemical or physical characterisation of a sample's natural radioactivity, the conversion of its natural luminescence into a radiometric age involves two laboratory experiments, in which the luminescence is monitored as a function of the exposure time $t$ [s] to (i) a source of constant radioactivity $\dot{D}$ [Gy s $\mathrm{s}^{-1}$, and (ii) a source of a constant temperature $T$ $[\mathrm{K}]$. The former experiment determines how fast does the luminescence signal grow under an artificial radiation source, and the latter (often skipped in routine sediment dating) quantifies the thermal stability of the dosimetric electron trap.

Although the observable rates of luminescence growth and decay in the laboratory are typically faster by a factor of $\sim 10^{10}$ than in nature, geological dating must assume that the kinetic parameters describing laboratory behaviour are fundamental physical characteristics of the material, that can be extrapolated over longer timescales and slower rates. Thus, the selection of a model for describing laboratory behaviour is more than critical for the correct and meaningful conversion of the natural luminescence intensities into equivalent ages. Even if a model produces an excellent fit to laboratory data, this cannot necessarily guarantee its successful extrapolation to geological timescales; at the same time, a model which does not fit laboratory data is even harder to evaluate, since it may further propagate this failure unpredictably, potentially yielding correct ages even though the model is inadequate. In this paper, we take a fresh look at the conventional 'status quo' models currently used to describe dose response and thermal sensitivity of feldspar IRSL. We further examine an interesting heuristic approach (the General-Order Kinetic model), and use a representative dataset to graphically illustrate the key differences between the models, and to quantify their relative successes and shortfalls. 


\section{Data and methods}

\subsection{Feldspar MET-pIRIR dataset}

The various models discussed in this paper were tested against data that was obtained using the multi-elevated temperature post-IR IRSL protocol (MET-pIRIR; Li and $\mathrm{Li}, 2011)$. This protocol retrieves five different IRSL signals measured at incrementally rising stimulation temperatures (50,100,150, 200 and $250{ }^{\circ} \mathrm{C}$ ), and typically exhibiting different thermal stabilities. The specific dataset used in our study, is taken from the work of $\mathrm{Li}$ and $\mathrm{Li}(2012 ; 2013)$, and is provided as a digital appendix for any future re-evaluation (see Supplementary Material). The data for each of the five post-IR signals (abbreviated MET-pIRIR ${ }_{x}$, where $x$ is the stimulation temperature) consists of a radiation-induced growth experiment (a single time-series, observed at a room temperature of $\sim 15^{\circ} \mathrm{C}$ ), and an isothermal decay experiment (four individual time-series, measured at temperatures of $300,320,240$ and $260{ }^{\circ} \mathrm{C}$, and fitted simultaneously).

\subsection{Fitting and smoothing procedures}

Nonlinear least-square fitting and estimation of errors was performed using the Isqnonlin and nlparci functions in Matlab. Trends in the fitting residuals (Fig. 1) and in the best-fit parameters (Fig. 3) were visualised using the locally weighted regression and smoothing (LOWESS) method of Cleveland (1979).

\subsection{Data visualisation}

An implicit tradition in modern OSL literature (e.g. Murray and Wintle, 2000) stipulates the presentation of radiation-induced growth in form of a 'dose response' curve, in which the luminescence light sum $L(t)$ varies as a function of the 'absorbed dose' $D=\dot{D} t$ (e.g. Fig. 1a-d). Conversely, isothermal decay experiments carried out on the same materials are typically visualised as $\log \left(L(t) / L_{0}\right)$ against time $t$ only (e.g. Murray and Wintle, 1999). In the present paper we use a slightly modified visualisation scheme (after Levy, 1961; 1991; Li and Li, 2013), in which the luminescence intensity $L(t)$ is always plotted against $\log (t)$ regardless of whether luminescence growth or decay are being explored. The specific benefits of such visualisations are: 
(i) Separation of data from interpretation. When luminescence $L(t)$ is plotted against the absorbed dose $D=\dot{D} t$, the x-axis unnecessary entangles a primary observation (irradiation time $t$ ) with a derived parameter (the dose rate $\dot{D}$ ), the latter incorporating multiple internal and external uncertainties (Bos et al., 2006; Guerin et al., 2012; Kadereit and Kreutzer, 2013; Boehnke and Harrison, 2014). Thus, a plot of $L(t)$ vs. $D$ technically becomes erroneous with every systematic revision of dose rate conversion factors, while a plot of $L(t)$ vs. $t$ will not only remain valid, but also be easier to re-analyse in the future. Furthermore, it is well-known that in materials suffering from athermal losses, delivery of the same dose at different irradiation rates leads to differential luminescence responses (e.g. Kars et al., 2008). Thus, showing luminescence response against an amalgamated variable which is the product of both time and dose rate $D=\dot{D} t$ leads to misapprehension of the dependence of luminescence build-up on laboratory dose rates (see Levy, 1961; 1991).

(ii) Visual informativeness: The processes of luminescence growth and decay are both governed by a fundamental rate term $\left[\mathrm{s}^{-1}\right]$, which drives each corresponding process towards a secular steady-state. Derivation of reliable kinetic parameters typically relies on data which is uniformly spaced across 3-4 orders of magnitude of time (e.g. Kars et al., 2008; Murray et al., 2009; Timar-Gabor et al., 2012). Thus, the use of a linear time axis may unfavourably compress information from a particular timescale, and lead to a visual misapprehension on fit quality, or the lack of experimental points to (dis)prove a certain model (compare Fig 1a-d with Fig. 1e-h, showing exactly the same data $L(t)$ but as a function of $D=\dot{D} t$ and $\log (t)$, respectively). The above problems are less likely to occur on a logarithmic time axis $\log (t)$, which not only grants easy comparison between similar processes occurring on different timescales, but also highlights regions where data is missing to properly constrain the model fitting

(iii) Uniformity for internal comparison: Visualisation of luminescence growth and decay as a function of $\log (t)$ allows a straightforward side-by-side comparison of the kinetic responses of the material to cumulative irradiation and heat, and facilitates both the detection and quantification of systematic departure from first order kinetics in both cases (see Section 3.3 and Fig. 2). Although the new 
standardised visualisation might be slightly difficult to compare to former studies (utilising the more familiar visualisations), we believe that this is a minor inconvenience outweighed by the benefits of internal intercomparison, and of an enhanced apprehension of model quality.

[Figure 1]

\section{Models and results}

\subsection{First-order (exponential) kinetics (1EXP)}

The growth of the IRSL light sum $L(t)$ [a.u.] in a feldspar exposed to a radioactive source may be described by a saturating exponential function:

$L(t) / L_{\max }=1-\exp \left(-\dot{D} t / D_{0}\right)$

(e.g. Balescu et al., 1997; $\mathrm{Li}$ and $\mathrm{Li}, 2012$ ) where $L_{\max }$ [a.u.] is the maximum luminescence light sum, $\dot{D}\left[\mathrm{~Gy} \mathrm{~s}^{-1}\right]$ the constant dose rate of the radioactive source, $t$ [s] the time, and $D_{0}[\mathrm{~Gy}]$ the characteristic dose. Similarly, the time-evolution of $L(t)$ [a.u.] under isothermal storage of the feldspar at temperature $T[\mathrm{~K}]$ may be described by a decaying exponential function:

$L(t) / L_{0}=\exp \left(-s e^{-E / k_{B} T} \cdot t\right)$

(e.g. Li et al., 1997; Murray et al., 2009), where $L_{0}$ [a.u.] is the initial IRSL light sum, $E[\mathrm{eV}]$ and $s\left[\mathrm{~s}^{-1}\right]$ the Arrhenius parameters (activation energy and the attempt-toescape frequency, respectively), $k_{B}\left[\mathrm{eV} \mathrm{K}^{-1}\right]$ Boltzmann's constant, and $t$ [s] the time as before.

Figures $1 \mathrm{a}$,e and $1 \mathrm{i}$ demonstrate the rather unsatisfactory fits of the 1EXP model to the irradiation response (top plots in Figs. 1a,e) and isothermal decay (Fig. 1i) of the MET-pIRIR 250 signal. Although for luminescence growth (top plots in Figs. $1 \mathrm{a}, \mathrm{e})$, the 1 EXP model explains $\sim 99 \%$ of the variance in the data, the residuals are not normally distributed over the time domain (bottom plot in Figs. 1a,e), stipulating the search for a better model. For the isothermal decay data, the overall $\mathrm{R}^{2}$ of 1 EXP $\left(\sim 85 \%\right.$ in Fig. 1i) is grossly overestimating the individual $R^{2}$ for each holding temperature, and thus evaluates this model as inappropriate. 


\subsection{Multi-exponential kinetics (mEXP)}

Observation of slow but steady growth of feldspar IRSL at high doses $\left(\dot{D} t>>D_{0}\right)$ is often empirically explained by a saturating exponential plus linear $(1 E X P+L I N)$ model:

$$
L(t)=L_{1}\left[1-\exp \left(-\dot{D} t / D_{0}\right)\right]+L_{2}\left[\dot{D} t / D_{0}\right]
$$

(e.g. Lai, 2010), where $L_{1}$ and $L_{2}$ [a.u.] are the saturating exponential and linear components, respectively (typically $L_{2}<<L_{1}$ ). Although such linear growth may be interpreted as a steady generation of new electron traps at a fixed rate (e.g. Levy, 1961), this phenomenon is more often viewed as the early expression of a second saturating exponential, corresponding to a different component or sub-population of the electron trap (Chen et al., 2001). Following the reasoning of signal break-up into individual components, the dose response of feldspar IRSL may be generalized to:

$$
L(t)=\sum_{1}^{m} L_{i}\left[1-\exp \left(-\dot{D} t / D_{0, i}\right)\right]
$$

where $m=2$ usually suffices (e.g. Thomsen et al., 2011; Buylaert et al., 2012), and where $L_{i}$ [a.u.] and $D_{0, i}$ [Gy] are the maximum light sum and characteristic dose of the $i$-th component. To justify the 2EXP model in quartz OSL, several working hypotheses have been put forth (Lowick et al., 2010; Berger and Chen, 2011; TimarGabor et al., 2012), but the phenomenon is still poorly understood (Wintle, 2011). From the chemical standpoint, the possibility of distinct dose-response components in feldspar is even more likely than in quartz (e.g. different $D_{0}$ values for each compositional end-member of feldspar; cf. Barré and Lamothe, 2010), however this conjecture is pending further proof.

Fits of $1 E X P+L I N$ and $2 E X P$ to the MET-pIRIR ${ }_{250}$ dataset are shown in Figs. $1 \mathrm{~b}-\mathrm{c}$ and 1f-g. From inspecting the residuals, it may be seen that 2EXP performs better due to one extra model parameter. However, the dataset is in fact insufficient to justify the break-up into the best-fit dose components $D_{0,1}=122 \pm 30$ Gy and $D_{0,2}=490 \pm 60 \mathrm{~Gy}$, as the $D_{0}$ values are too closely spaced (see review by Istratov and Vyvenko, 1999). Interestingly, neither of the above values, nor the $D_{0}=244 \pm 9$ Gy from $1 E X P+L I N$, overlap with the baseline value of $D_{0}=315 \pm 8$ Gy, retrieved by not necessarily the correct, yet the simplest $1 E X P$ model. 
Switching to multi-exponential description of isothermal loss, we start with the model of Jain et al. (2012), who expressed the thermal loss of trapped charges via a quantum mechanical tunnelling from the excited state of the electron trap. In the resulting multi-exponential system (where first-order loss occurs only for a fixed electron-hole separation distance), the decrease of luminescence intensity with progressive isothermal storage can be approximated by:

$L(t) / L_{0}=\exp \left\{-\rho^{\prime}\left[\ln \left(1+1.8 \cdot s e^{-E / k_{B} T} \cdot t\right)\right]^{3}\right\}$

(Kitis and Pagonis, 2013), where $L_{0}$ [a.u.] is the initial intensity, and $\rho^{\prime}$ the scaled density of the nearest-neighbour distribution of holes. Notably, Eq. (5) reduces to the athermal tunnelling model of Huntley (2006) upon the substitution $E=0$, thereby generalising Huntley's model to thermally-assisted processes. The fit of Eq. (5) to the MET-pIRIR 250 data is shown in Fig. 1i, with narrowly constrained parameters and no appreciable time dependence or structure in the residuals.

A different multi-exponential approach was taken by $\mathrm{Li}$ and $\mathrm{Li}$ (2013), who assumed that trapped electrons are thermally activated to discrete and exponentially distributed energy levels below the conduction band (known as band tail states, Poolton et al., 2002; 2009). Envisaging a spatial distribution where each electron trap is associated with only one band-tail energy level above it, Li and Li (2013) expressed the overall thermal decay of luminescence as:

$L(t) / L_{0}=\int_{0}^{E} e^{-E_{b} / E_{u}} e^{-s e^{-\left(E-E_{b}\right) / k_{B} T} \cdot t} d E_{b}$

where $L_{0}$ [a.u.] is the initial intensity, and $E_{u}[\mathrm{eV}]$ is the Urbach band-tail width. Eq. (6a) reduces to Eq. (2) for $E_{b} \rightarrow 0$, and thereby qualifies as its logical extension. To derive a convenient approximation for Eq. (6a) for data fitting, we introduce $b=E_{b} / k_{B} T, u=E_{u} / k_{B} T$, and $\tau=t \cdot s e^{-E / k_{B} T}$, and rewrite Eq. (6a) as:

$L(t) / L_{0}=k_{B} T \int_{0}^{b_{0}} e^{-b / u} e^{-\tau e^{b}} d u$

To bypass tedious numerical integration, this paragraph derives a convenient analytical approximation for Eq. (6b), which can be easily implemented in common curve-fitting software. We begin by a change of variables $w=e^{b}$ and rearrange the latter into $b=\ln w, \quad d b=d w / w$, to obtain: 
$L(t) / L_{0}=k_{B} T \int_{1}^{w_{0}} w^{-1-1 / u} e^{-\tau w} d w=k_{B} T \tau^{1 / u} \int_{\tau}^{\tau w_{0}} x^{-1-1 / u} e^{-x} d x=k_{B} T \tau^{1 / u}\left\{\Gamma(-1 / u, \tau)-\Gamma\left(-1 / u, \tau e^{b_{0}}\right)\right\}$

where $\Gamma$ is the upper incomplete gamma function. Back-substitution of the original variables, and omission of the negligible second $\Gamma$ term results in:

$L(t) / L_{0}=k_{B} T\left(t \cdot s e^{-E / k_{B} T}\right)^{k_{B} T / E_{u}} \Gamma\left(-k_{B} T / E_{u}, t \cdot s e^{-E / k_{B} T}\right)$

Eq. (6d) is the desired approximation of Eq. (6a). The fit of Eq. (6d) to the METpIRIR $_{250}$ data is shown in Fig. 1j, displaying a reasonable fit, but with an undesirable non-uniform distribution of the residuals.

\subsection{General order kinetics (GOK)}

The familiar first-order description of luminescence growth and decay (Eqs. 12) may be generalised to:

$$
\begin{aligned}
& L(t) / L_{\max }=1-\exp (-t / \tau) \\
& L(t) / L_{\max }=\exp (-t / \tau)
\end{aligned}
$$

where $L(t) / L_{\max }$ is the normalized luminescence light sum [a.u.], $t$ [s] is time, and $\tau$ [s] a time constant. To depart from first order kinetics, we follow Whitehead et al. (2009) by introducing a time-dependent $\tau(t)=\tau_{0}+c t$, where $c>0$ is a kinetic order modifier, and rewrite $t / \tau$ as:

$$
t / \tau=\int_{0}^{t} \frac{1}{\tau(t)} d t=\int_{0}^{t} \frac{1}{\tau_{0}+c t} d t=\frac{1}{c} \ln \left(1+\frac{c t}{\tau_{0}}\right)
$$

To obtain the general-order expressions, we insert Eq. (9) into Eqs. (7-8), and make the additional substitutions $\tau_{0}=D_{0} / \dot{D}$ for radiation-induced growth, and $\tau_{0}=s^{-1} \exp \left(E / k_{B} T\right)$ for thermally-activated decay, to obtain:

$$
\begin{aligned}
& L(t) / L_{\max }=1-\left(1+\left(\dot{D} / D_{0}\right) c t\right)^{-1 / c} \\
& L(t) / L_{\max }=\left(1+s e^{-E / k_{B} T} c t\right)^{-1 / c}
\end{aligned}
$$

Note that for $c \rightarrow 0$, the new Eqs. (10-11) asymptotically reduce to Eqs. (1-2), but as $c$ increases they progressively deviate from first-order behaviour (Fig. 2). Note that Eq. (11) has been already used to fit isothermal decay of luminescence in quartz (Ankjærgaard et al. 2013; Wu et al., this issue), and although, to the best of our knowledge, Eq. (10) is unprecedented within luminescence dosimetry literature, it appears as a perfectly valid and logical counterpart of Eq. (11). 
[Figure 2]

To further explore the placement of Eqs. (10-11) within the context of generalorder kinetics, we differentiate both equations with respect to time, assume the standard proportionality between luminescence and trapped charge $(L(t) \rightarrow n$, $\left.L_{\max } \rightarrow N\right)$, and make a convenient variable replacement $(\alpha=c+1, \beta=c+1)$ to translate Eqs. (10-11) into:

$\frac{d}{d t}\left(\frac{n}{N}\right)=\frac{\dot{D}}{D_{0}}\left(1-\frac{n}{N}\right)^{\alpha}$

$\frac{d}{d t}\left(\frac{n}{N}\right)=-s e^{-E / k_{B} T}\left(\frac{n}{N}\right)^{\beta}$

in which $n$ is the number of electrons trapped in $N$ traps of a certain type [both a.u.], and $\alpha$ and $\beta$ are the kinetic orders [a.u.] of the electron trapping and detrapping reactions, respectively.

The effect of the unitless kinetic orders $\alpha$ and $\beta$ on the luminescence growth and decay is graphically shown in Fig. 2 and discussed below. In first-order kinetics $(\alpha \equiv 1, \beta \equiv 1)$ the growth and decay rates of luminescence are always independent of the amount of trapped charge $n / N$ (thus justifying the definition of a trap lifetime). Conversely, in higher order kinetics $(\alpha>1, \beta>1)$ reaction do depend on trap occupation, and always progress at slower-than-exponential rates. This may be mathematically appreciated by looking at Eqs. 12-13, where the fractions of empty and filled electron traps $(1-n / N$ and $n / N$, respectively), always smaller than unity, are both further diminished when raised to a power of $\alpha>1, \beta>1$.

From the physical standpoint, the progressive slowdown of reaction rates in systems which are nearly empty or borderline their full capacity (i.e. close to the boundary conditions) is both understandable and predictable. Specifically, the slower-than-exponential electron detrapping $(\beta>1)$ has been often considered in the luminescence literature (e.g. Wise, 1951; May and Partridge, 1964; Rasheedy, 1993), and explained in terms of electron retrapping or distance-dependent probabilities. Conversely, the slower-than-exponential trapping of electrons $(\alpha>1)$ in a confined volume due to the gradual build-up of Coulomb repulsive forces is a wellstudied phenomenon in field-effect transistors (e.g. Sune et al., 1990; Williams, 
1992). Buildup of Coulomb forces, and the presently overlooked effects of possible charge disequilibrium within irradiated crystals, are both subjects of increasing interest within the luminescence dating community, stipulating new research directions being currently underway (J.-P. Buylaert, pers. comm.).

While a further physical validation of Eqs. (12-13) remains outside of the scope of the present work, we note that their superposition results in:

$\frac{d}{d t}\left(\frac{n}{N}\right)=\frac{\dot{D}}{D_{0}}\left(1-\frac{n}{N}\right)^{\alpha}-s e^{-E / k_{B} T}\left(\frac{n}{N}\right)^{\beta}$

which for $\alpha \equiv \beta \equiv 1$ reduces to the familiar description of thermoluminescence systems (Christodoulides et al., 1971), and for $\alpha, \beta \geq 1$ serves as its logical extension for more complex (i.e. slowed-down) behaviour.

The fits of Eqs. (10-11) to the MET-pIRIR 250 growth and decay are shown in Figs. $1 \mathrm{~d}, \mathrm{~h}$ and $1 \mathrm{k}$, respectively. Interestingly, the $D_{0}$ 's recovered by the 1 EXP and GOK models are indistinguishable; from this perspective, GOK is the only extended model which introduces a further complexity without affecting the primary response variable $\left(D_{0}\right)$ as obtained from the least sophisticated model (Eq. 1). For the isothermal holding, the GOK model fits the experimental dataset equally well as mEXP tunnelling, further exhibiting the narrowest confidence intervals.

\section{Discussion}

The best-fit kinetic parameters for the MET-pIRIR 250 signal from Fig. 1 are summarised in Fig. 3 (filled circles) and further supplemented by the best-fit parameters from the other four MET-pIRIR signals (MET-pIRIR ${ }_{50}-$ MET-pIRIR 200 ). Starting with the radiation-induced growth dataset (Fig. 3a-d), it seems that irrespective of the chosen model, $D_{0}$ appears to be anti-correlated with the MET stimulation temperature, yielding progressively smaller $D_{0}$ 's for the least fading

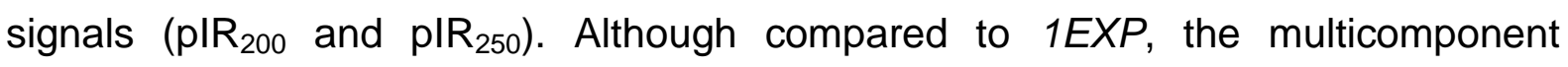
$1 E X P+L I N$ and the 2EXP models appear as plausible fits on the typical 'doseresponse' curves (Figs. 1b-c), they appear as unconstrained over-fitting artefacts, lacking model verification in their high-dose domain (clearly seen as the unconstrained model predictions in Figs. 1f-g), and therefore raising further concern for their use for predicting minimum ages or thermal closure ages, where the steady- 
state response of the system becomes a crucial consideration. The GOK model looks promising not only because it fits the experimental data best with a minimum of model parameters, but also because it retains the same $D_{0}$ 's as the simplest $1 E X P$ model; however, the validity of this approach both in the high-dose region and within natural environments, is clearly subject to further investigation.

For the isothermal decay dataset (Figs. 3e-h), the 1EXP model seems absolutely inadequate. In the bandtail mEXP, tunnelling mEXP and GOK models, there is a clear correlation between a single response variable $\left(E_{u}, \log _{10} \rho^{\prime}\right.$ or $\left.c\right)$ with the post-IR stimulation temperature, which the Arrhenius parameters assuming $E$ and $s$ remain semi-constant. However, a high covariance between $E$ and $E_{u}$ in the bandtail mEXP points to an ill-conditioned fit, to be addressed though a reduction of the number of parameters (e.g. assuming $E, E_{u}$ or $s$ to be constant; cf. $\mathrm{Li}$ and $\mathrm{Li}, 2013)$. The tunnelling mEXP model yields kinetic parameters that are supported in literature, including a familiar $s$ value in the range $10^{12}-10^{14} \mathrm{~s}^{-1}$, and $E \sim 1.4 \mathrm{eV}$ corresponding to the optical energy of the excited state; how these results apply to pIRIR signals involving transitions through band tail states is a separate question worth investigating (see Jain et al., this volume). The GOK model yields $E \sim 1.3 \mathrm{eV}$ and $s \sim 10^{9}$, both of which are anomalously low compared to familiar literature values ( $\mathrm{Li}$ et al., 1997; Murray et al., 2009; Li and Li, 2013). While the extrapolation of these kinetic parameters to geological timescales seems to be successful (Guralnik et al., subm.), additional effort is required to understand whether these best-fit parameters fold in the initial experimental conditions of the explored systems (cf. Rasheedy, 1993).

[Figure 3]

Although heuristic, the proposed GOK model offers a convenient and selfconsistent alternative to the more established multi-exponential analysis, yielding plausible fits to experimental data with a comparable (or fewer) number of model parameters, and a well-known physical reasoning. Although the dataset is too small to allow a meaningful statistical inference $(n=5)$, it is worthwhile to note that the kinetic orders of dose response (Fig. 3d) and isothermal holding (Fig. 3h) appear to be pairwise correlated. This suggests that a particular system's departure from first- 
order kinetics (Fig. 2) may be manifested in mirroring electron trapping and detrapping processes. This observation further justifies the proposed uniform visualisation $L(t)$ vs. $\log (t)$ for both dose response and isothermal holding experiments, as it may help identify and correlate the departure from first-order kinetics in both these processes. Furthermore, the hypothesized correlation $\alpha \propto \beta$ invites to consider a continuous multi-exponential fitting (Eqs. 5 or 6) for the description of dose response in feldspar, which is currently only modelled by a finite and usually small number of dose-response components.

The present study has focused on evaluating the different feldspar models against a set of laboratory experiments, where the rates of electron trapping and detrapping are roughly $\sim 10^{10}$ times faster than in typical natural settings. The next desirable step would be to test these models in natural conditions, where there is a maximum number of independent constraints on the sedimentation age, the duration of surface exposure, or the thermal regime. Noticeable mismatches between laboratory and natural dose response curves (Chapot et al., 2012; Zander and Hilgers, 2013) stipulate the evaluation of all models in their high-dose (steady-state) region, not regularly covered by standard laboratory measurements (Fig. 1e-h). Better characterisation of the high-dose region would also be beneficial for minimum age reporting (e.g. Joordens et al., 2014) or for thermochronological interpretation (Guralnik et al., 2013). In particular, the applicability of the general-order kinetics (GOK) model to natural conditions seems very promising, and will be reported elsewhere (Wu et al., this volume; Ankjærgaard et al., this volume; Guralnik et al., under review).

\section{Conclusions}

This paper reviewed common models describing dose response and isothermal decay in feldspar IRSL dating, and introduced a self-consistent general order kinetic model which produces good fits to laboratory data. As a first step towards proper model evaluation and intercomparison, we promote the use of a logarithmic time axis for the visualisation of both dose response and isothermal holding experiments. As a second step, we have demonstrated that representative feldspar IRSL data cannot be adequately described by first-order kinetics, while some of the common multi-exponential approaches are seen to suffer from 
covariated (and thus potentially non-identifiable) parameters. The proposed general order kinetics model captures both the laboratory dose response and isothermal decay of feldspar IRSL well, but may only be a gross mathematical simplification of actual physical processes; nevertheless it is a promising path towards methodological standardisation, stipulating further basic research and comparative model verification in well-constrained geological environments.

\section{Acknowledgements}

This work was supported by the Swiss National Foundation grants 200021127127 (FH and BG) and PZ00P2-148191 (PGV), and has benefitted from stimulating discussions with David Sanderson, Christina Ankjærgaard and Sally Lowick.

\section{References}

Ankjærgaard, C., Jain, M., Wallinga, J., 2013. Towards dating Quaternary sediments using the quartz Violet Stimulated Luminescence (VSL) signal. Quat. Geochron. 18, 99-109.

Ankjærgaard, C., Guralnik, B., Porat, N., Heimann, A., Jain, M., Wallinga, J., (this volume). Violet stimulated luminescence: geo- or thermochronometer? Radiat. Meas.

Balescu, S., Lamothe, M., Lautridou, J.P., 1997. Luminescence evidence for two Middle Pleistocene interglacial events at Tourville, northwestern France. Boreas, 26, 61-72.

Barré, M., Lamothe, M., 2010. Luminescence dating of archaeosediments: a comparison of K-feldspar and plagioclase IRSL ages. Quat. Geochron. 5, 324-328.

Berger, G.W., 1990. Regression and error analysis for a saturating-exponential-plus-linear model. Ancient TL, 8, 23-25.

Berger, G.W., Chen, R., 2011. Error analysis and modelling of double saturating exponential dose response curves from SAR OSL dating. Ancient TL 29, 9-14.

Boehnke, P., Harrison, T. M., 2014. A meta-analysis of geochronologically relevant half-lives: what's the best decay constant? Int. Geol. Rev. 56, 905-914.

Bos, A.J.J., Wallinga, J., Johns, C., Abellon, R.D., Brouwer, J.C., Schaart, D.R., Murray, A.S., 2006. Accurate calibration of a laboratory beta particle dose rate for dating purposes. Radiat. Meas. 41, 1020-1025.

Buylaert, J.P., Jain, M., Murray, A.S., Thomsen, K.J., Thiel, C., Sohbati, R., 2012. A robust feldspar luminescence dating method for Middle and Late Pleistocene sediments. Boreas 41, 435-451.

Chapot, M.S., Roberts, H.M., Duller, G.A.T., Lai, Z.P., 2012. A comparison of natural-and laboratory-generated dose response curves for quartz optically stimulated luminescence signals from Chinese Loess. Radiat. Meas. 47, 1045-1052.

Chen, G., Murray, A.S., Li, S.H., 2001. Effect of heating on the quartz dose-response curve. Radiat. Meas. 33, 59-63.

Christodoulides, C., Ettinger, K.V., Fremlin, J.H., 1971. The use of TL glow peaks at equilibrium in the examination of the thermal and radiation history of materials. Modern Geology 2, 275-280.

Cleveland, W.S., 1979. Robust locally weighted regression and smoothing scatterplots. J. Am. Stat. Assoc. 74, 829-836.

Guérin, G., Mercier, N., Adamiec, G., 2011. Dose-rate conversion factors: update. Ancient TL, 29, 5-8.

Guibert, P., Vartanian, E., Bechtel, F., Schvoerer, M., 1996. Non-linear approach of TL response to dose: polynomial approximation. Ancient TL, 14, 7-14.

Guralnik, B., Jain, M., Herman, F., Murray, A.S., Valla, P.G., Ankjærgaard, C., Lowick, S.E., Preusser, F., Chen, R., Kook, M.H., Rhodes, E.J. (subm.). OSL-thermochronology of bedrock feldspar reveals subQuaternary thermal histories of near- surface rocks.

Guralnik, B., Jain, M., Herman, F., Paris, R.B., Harrison, T.M., Murray, A.S., Valla, P.V., Rhodes, E.J., 2013. Effective closure temperature in leaky and/or saturating thermochronometers. Earth Planet. Sci. Lett. 384, 209-218. 
Huntley, D.J., 2006. An explanation of the power-law decay of luminescence. J. Phys. Cond. Matt. 18, 13591365.

Hütt, G., Jaek, I., Tchonka, J., 1988. Optical dating: K-feldspars optical response stimulation spectra. Quat. Sci. Rev. 7, 381-385.

Istratov, A.A., Vyvenko, O.F., 1999. Exponential analysis in physical phenomena. Rev. Sci. Inst. 70, 12331257.

Jain, M., Guralnik, B., Andersen, M.T., 2012. Stimulated luminescence emission arising from localized recombination within randomly distributed defects. J. Phys. Cond. Mat., in press.

Joordens, J.C., d'Errico, F., Wesselingh, F. P., Munro, S., de Vos, J., Wallinga, J., Ankjærgaard, C., Reimann, T., Wijbrans, J.R., Kuiper, K.F., Mücher, H.J., Coqueugniot, H., Prié, V., Joosten, I., van Os, B., Schulp, A.S., Panuel, M., van der Haas, V., Lustenhouwer, W., Reijmer J.J.G., Roebroeks, W., 2014. Homo erectus at Trinil on Java used shells for tool production and engraving. Nature, doi:10.1038/nature13962

Kadereit, A., Kreutzer, S., 2013. Ris $\emptyset$ calibration quartz-A challenge for $\beta$-source calibration. An applied study with relevance for luminescence dating. Measurement 46, 2238-2250.

Kars, R.H., Wallinga, J., Cohen, K.M., 2008. A new approach towards anomalous fading correction for feldspar IRSL dating - tests on samples in field saturation. Radiat. Meas. 43, 786-790.

Kitis, G., Pagonis, V., 2013. Analytical solutions for stimulated luminescence emission from tunneling recombination in random distributions of defects. J. Lumin. 137, 109-115.

Lai, Z. P., Stokes, S., Bailey, R., Fattahi, M., Arnold, L., 2003. Infrared stimulated red luminescence from Chinese loess: basic observations. Quat. Sci. Rev. 22, 961-966.

Levy, P.W., 1961. Color centers and radiation-induced defects in Al2O3. Phys. Rev. 12, 1226-1233.

Levy, P.W., 1991. Radiation damage studies on non-metals utilizing measurements made during irradiation. J. Phys. Chem. Solids 52, 319-349.

Li, B., Jacobs, Z., Roberts, R.G., Li, S.H., 2014. Review and assessment of the potential of post-IR IRSL dating methods to circumvent the problem of anomalous fading in feldspar luminescence. Geochronometria, 1-24.

Li, B., Li, S.H., 2008. Investigations of the dose-dependent anomalous fading rate of feldspar from sediments. J. Phys. D. Appl. Phys. 41, 225502.

Li, B., Li, S.-H., 2011. Luminescence dating of K-feldspar from sediments: A protocol without anomalous fading correction. Quat. Geochron. 6, 468-479.

Li, B., Li, S.H., 2012. Luminescence dating of Chinese loess beyond 130 ka using the non-fading signal from Kfeldspar. Quat. Geochron. 10, 24-31.

Li, B., Li, S.H., 2013. The effect of band-tail states on the thermal stability of the infrared stimulated luminescence from K-feldspar. J. Lumin., 136, 5-10.

Li, S.H., Tso, M.Y.W., Wong, N.W., 1997. Parameters of OSL traps determined with various linear heating rates. Radiat. Meas. 27, 43-47.

Lowick, S.E., Preusser, F., Wintle, A.G., 2010. Investigating quartz optically stimulated luminescence doseresponse curves at high doses. Radiat. Meas. 45, 975-984.

McKeever, S.W.S., Chen, R., 1997. Luminescence models. Radiat. Meas. 27, 625-661.

Murray, A.S., Buylaert, J.P., Thomsen, K.J., Jain, M., 2009. The effect of preheating on the IRSL signal from feldspar. Radiat. Meas. 44, 554-559.

Murray, A.S., Wintle, A.G., 1999. Isothermal decay of optically stimulated luminescence in quartz. Radiat. Meas. 30, 119-125.

Murray, A.S., Wintle, A.G., 2000. Luminescence dating of quartz using an improved single-aliquot regenerative-dose protocol. Radiat. Meas. 32, 57-73.

Poolton, N.R.J., Kars, R.H., Wallinga, J., Bos, A.J.J., 2009. Direct evidence for the participation of band-tails and excited-state tunnelling in the luminescence of irradiated feldspars. J. Physics Cond. Matt. 21, 485-505.

Sohbati, R., Murray, A.S., Jain, M., Buylaert, J.-P., Thomsen, K.J., 2011. Investigating the resetting of OSL signals in rock surfaces. Geochronometria 38, 249-258.

Rasheedy, M.S., 1993. On the general-order kinetics of the thermoluminescence glow peak. J. Phys. Cond. Matt. 5, 633-636.

Sune, C.T., Reisman, A., Williams, C.K., 1990. A new electron-trapping model for the gate insulator of insulated gate field-effect transistors. J. Electron. Mat. 19, 651-655.

Thomsen, K.J., Murray, A.S., Jain, M., 2011. Stability of IRSL signals from sedimentary K-feldspar samples. Geochronometria, 38, 1-13.

Timar-Gabor, A., Vasiliniuc, Ş., Vandenberghe, D.A.G., Cosma, C., Wintle, A.G., 2012. Investigations into the reliability of SAR-OSL equivalent doses obtained for quartz samples displaying dose response curves with more than one component. Radiat. Meas. 47, 740-745.

Whitehead, L., Whitehead, R., Valeur, B., Berberan-Santos, M., 2009. A simple function for the description of near-exponential decays: the stretched or compressed hyperbola. Am. J. Phys. 77, 173-179.

Williams, C.K., 1992. Kinetics of trapping, detrapping, and trap generation. J. Electron. Mat. 21, 711-720. 
Wintle, 2010. Future directions of luminescence dating of quartz. Geochronometria 37, 1-7.

Wu, T.-S., Jain, M., Guralnik, B., Murray, A.S., Chen, Y.-G. (subm. to LED2014 proceedings). Evaluation of Quartz OSL as a thermochronometer for unraveling recent cooling rates in the Hsuehshan Range (Central Taiwan).

Zander, A., Hilgers, A., 2013. Potential and limits of OSL, TT-OSL, IRSL and pIRIR 290 dating methods applied on a Middle Pleistocene sediment record of Lake El'gygytgyn, Russia. Clim. Past 9, 719-733.

\section{Figure captions}

Figure 1: Irradiation-response (a-h) and isothermal decay (i-l) of feldspar METpIRIR 250 signal (filled circles on top panels) as best-fitted by the various models discussed in the text (lines with 95\% confidence interval on top panels), with quoted best-fit parameters and goodness-of-fit. Fitting residuals and their trends (dots and lines on the bottom panels) were obtained by LOWESS (locally-weighted scatterplot smoothing; Cleveland, 1979).

Figure 2: Radiation induced growth (a) and isothermal decay (b) for different kinetic orders in the range 1-5, obtained via Eqs. (10) and (11) upon the substitutions $c=\alpha-1$ and $c=\beta-1$, respectively.

Figure 3: Cross-model summary of the best-fitting parameters for the MET-pIRIR 250 signal from Fig. 1 (filled circles), alongside best-fitting parameters for the four lower temperature MET-pIRIR signals, given as Supplementary Data (hollow circles). 
Figure 2

a) Radiation-induced growth

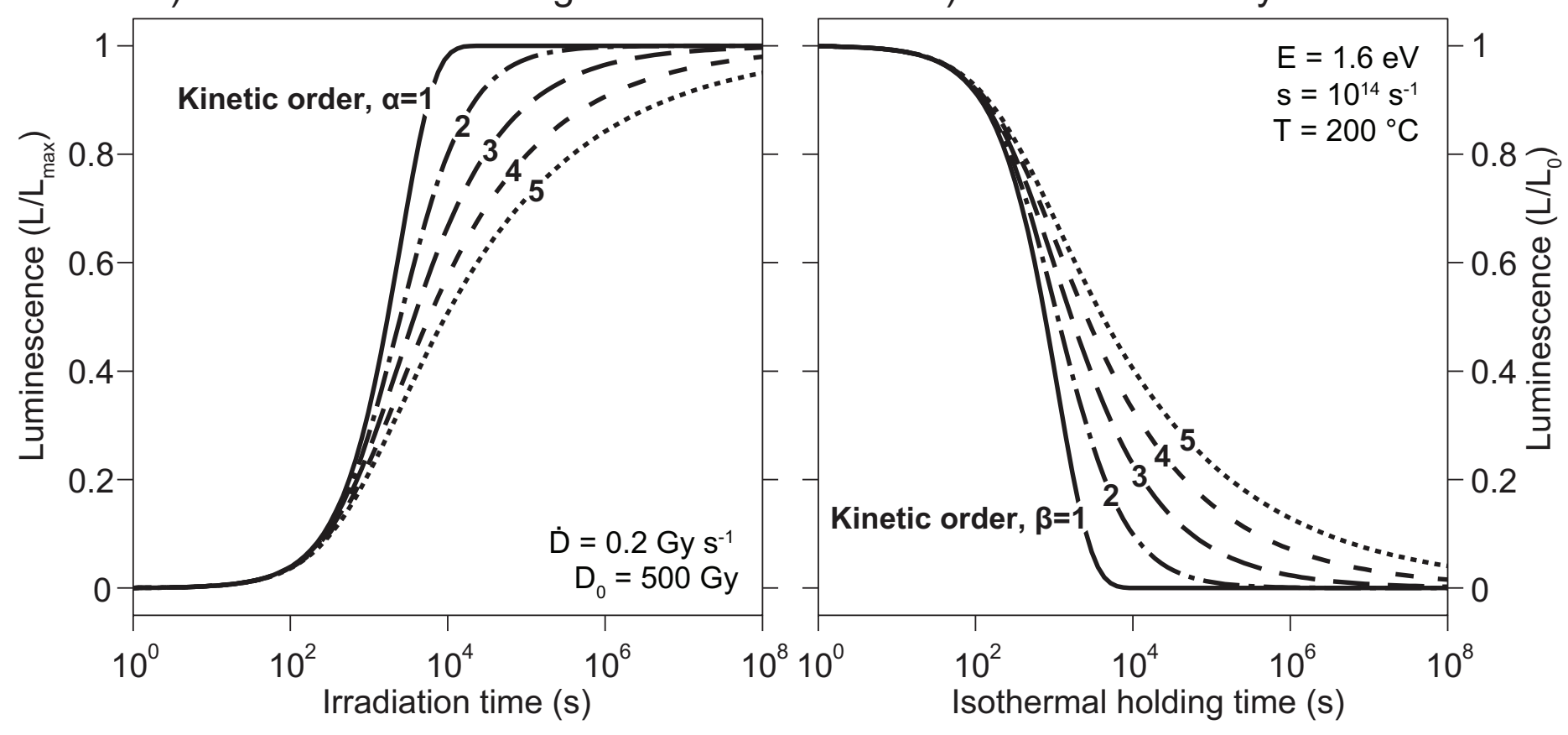



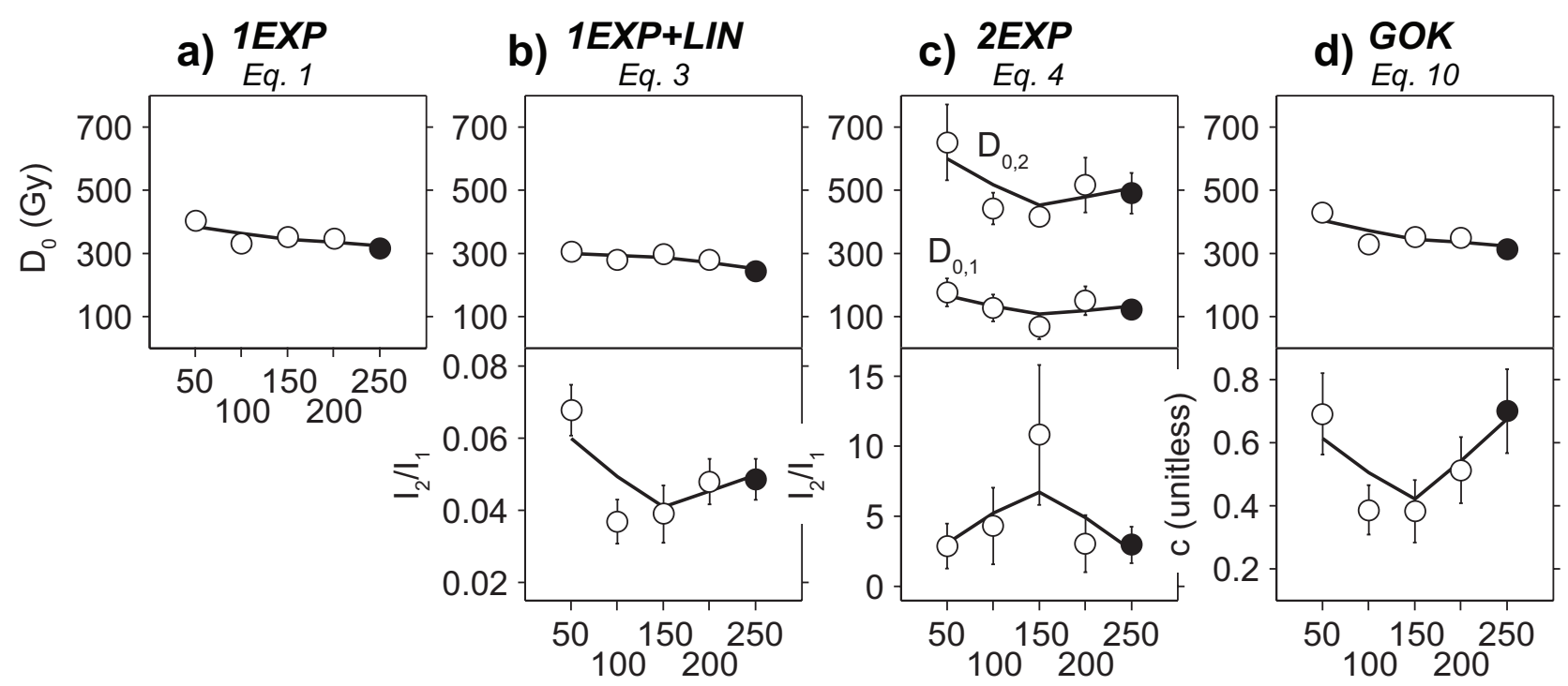

MET-pIR stimulation temperature $\left({ }^{\circ} \mathrm{C}\right)$

Isothermal decay
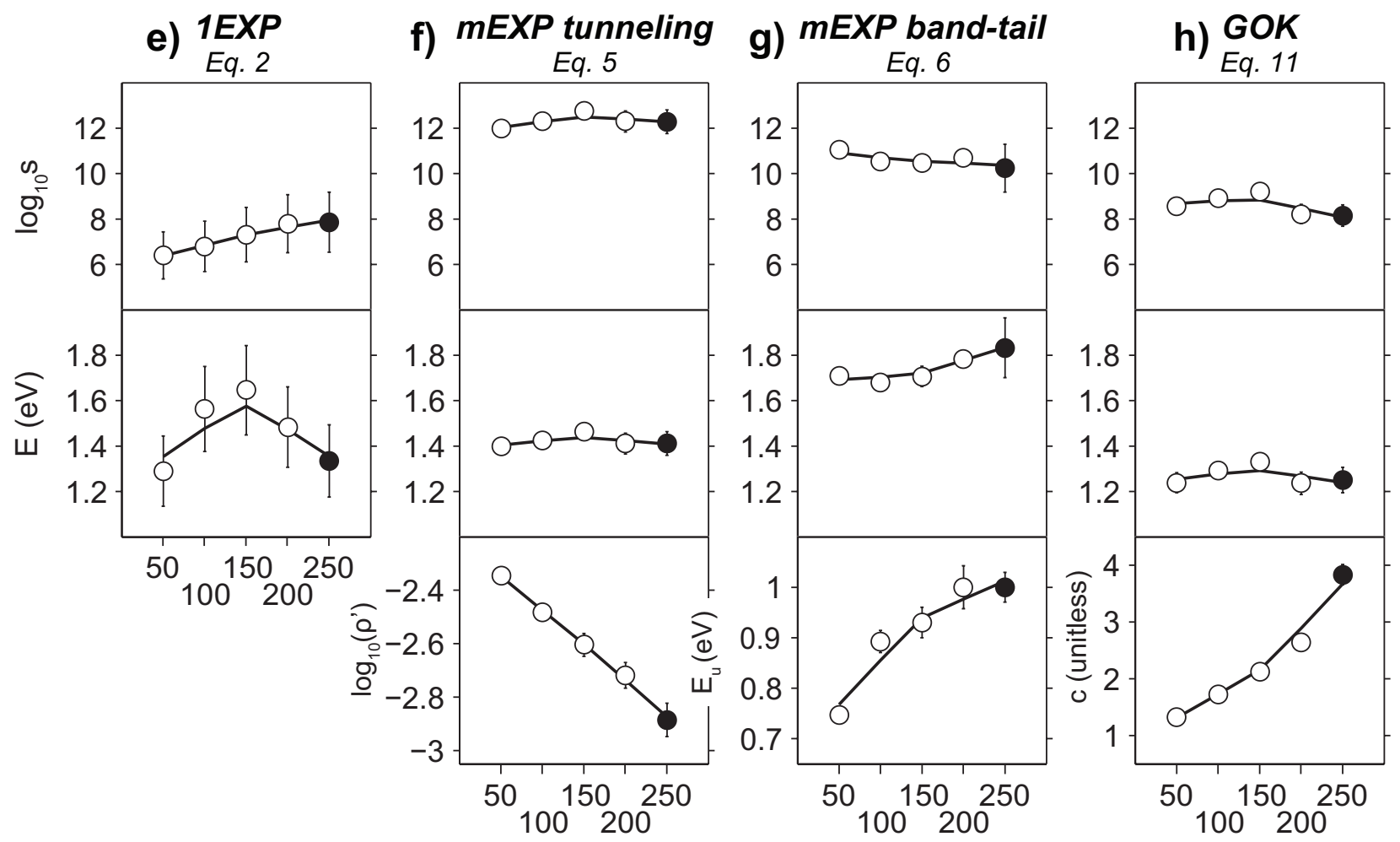

MET-pIR stimulation temperature $\left({ }^{\circ} \mathrm{C}\right)$ 